the MCT diet; drowsiness occurred in $25 \%$ at the introduction of the diet. Loss of ketosis occurred during the prodramal phase of an intercurrent illness and was often accompanied by an increase in the frequency of seizures. Two children under one year of age showed no increase in weight, length or head circumference during a six month period on the diet. (Schwartz R H et al. Ketogenic diets in the treatment of epilepsy: Short term clinical effects. Dev Med Child Neurol April 1989; 31:145-151).

\title{
METABOLIC EFFECTS OF KETOGENIC DIETS
}

The results of 24 metabolic profiles performed on 55 epileptic children receiving the classical ketogenic diet, the MCT diet, a modified MCT diet, and normal diets are reported from the University Department of Paediatrics, John Radcliffe Hospital, Oxford, England. The clinical effects of the diets are reported in the previous paper. All three therapeutic diets improved the control of epilepsy and induced a significant increase in the concentrations of blood aceto-acetate and 3-hydroxybutyrate, the greatest elevation being seen in patients on the classical diet (4:1). The pre- and post-prandial blood ketone levels with the classical diet reached a mean of $2.6 \mathrm{mmol} / \mathrm{L}$ before breakfast and $4 \mathrm{mmol} / \mathrm{L}$ before supper. The three ketogenic diets led to a buildup of ketone body concentrations during the day, reaching maximum levels in the afternoon. This was in contrast to the normal diet which led to slightly higher levels in the morning fasting samples. The Ketostix reagent strip test for urinary ketone bodies reflected these changes and showed higher levels in the afternoon and lower levels in morning samples. Children between 5 and 10 years of age showed the highest blood ketone levels. Levels of blood glycerol were highest while fasting and lowest after meals. Despite the high fat content of the diets none of the concentrations of plasma cholesterol, high density lipoproteins, low density and very low density lipoproteins was significantly raised in any of the therapeutic diet groups. Hypoglycemia was not documented in any patient at any time but blood concentrations of pyruvate were significantly lower. Lower blood levels of alanine occurred on all three diets, the most marked difference being in children receiving the classical diet. The remaining plasma amino acid concentrations tended to be lowest on the classical diet but other than alanine values, the mean concentrations of individual amino acids on the three diets failed to show any significant change. Plasma insulin concentrations corresponded to the blood glucose profiles, showing elevations after each meal, the highest levels occurring with the normal and modified MCT diet and the lowest responses occurring with the classical ketogenic diet. The mean plasma concentrations of sodium, potassium, chloride and bicarbonate did not differ significantly between the four diets, and plasma urea, creatinine, calcium, phosphate, total protein, albumin and bilirubin levels were also similar. Plasma uric acid levels were higher on all three ketogenic diets with the highest increase on the MCT diet. The mechanism of action of the ketogenic diet was not determined. (Schwartz R M et al. Metabolic effects of three ketogenic diets in the treatment of severe epilepsy. Dev Med Child Neurol April 1989; $31: 152-160)$.

COMMENT. The above two studies performed at the John Radcliffe Hospital in Oxford and including patients from the pediatric practice of Dr. B.D. Bower have reconfirmed the efficacy of the ketogenic diet in the management of intractable epilepsy in children under 15 years. The 
classical diet was more acceptable than the MCT diet, being equally effective and better tolerated by most patients. The authors were unable to document any significant changes in blood lipid profiles in the short term study, and the theoretical risks of inducing ischemic heart disease appeared to be outweighed by the benefit of the diets in controlling disabling seizures. With the increasing concern and attention to cholesterol and heart disease, however, this aspect of treatment must be followed carefully and patients with a family history of hypercholesterolemia or ischemic heart disease should probably be excluded from the ketogenic treatment program.

Balance studies are needed to determine the effect of the ketogenic diet on body water and electrolytes. In a balance study performed at the Mayo Clinic (Millichap JG, Jones JD. Acid-base, electrolyte, and amino-acid metabolism in children with petit mal. Etiologic significance and modification by anticonvulsant drugs and the ketogenic diet. Epilepsia 1964; 5:239-255). We found a decrease in the blood pH, $\mathrm{POO} 2$, and standard bicarbonate during short ketogenic periods. The urinary excretion of electrolytes was increased and particularly that of calcium, magnesium and sodium, and the balance of sodium, potassium, calcium, magnesium, phosphorus and nitrogen were negative. The excretion of alphaaminonitrogen was reduced, the excretion of free amino acids was variable, and the level of leucine in the serum was elevated. Fluid intake and urine output were reduced and the fall in body weight was rapid and marked in the initial week of treatment. The total lipids, fatty acid and cholesterol in the serm were increased but not significantly during the ketogenic diet period; they became elevated significantly when carbohydrates and the antiketogenic diet were reintroduced. The anticonvulsant action of the ketogenic diet was unrelated to diuresis, independent of acidosis, and was correlated with an increased urinary excretion and a negative balance of sodium and potassium. Calcium supplements are usually advised with the ketogenic diet and in addition magnesium supplements should probably be included. The ketogenic dietary therapy of childhood epilepsies deserves further attention from pediatric neurologists. Assurance of parental and patient cooperation is essential as well as skilled dietetic advice and follow-up. The classical diet is probably more acceptable and has less gastrointestinal side-effects than the MCT diet; a lower and more palatable ratio $(3: 1)$ than that used in the Oxford study is usually sufficient and effective. If the diet is continued for long periods, consultation with a specialist in lipid metabolism should be obtained and ultrasound of the liver ordered to exclude fatty infiltration of the liver. (See Ped Neur Briefs, April 1988; $\underline{2: 28)}$

\section{VASCULAR DISORDERS}

\section{ISCHEMIC STROKE IN CHILHOOD}

Juvenile ischemic cerebral vascular disease was studied over a 15 year period in 34 patients in the Department of Neurosurgery, Neurological Institute, Tokyo Women's Medical College, Tokyo, Japan. Intracranial occlusions were attributed to cerebral thrombosis or embolism in 23, and to 